\title{
Association between OGG1 Ser326Cys and APEX1 Asp148Glu polymorphisms and breast cancer risk: a meta-analysis
}

Qiliu Peng ${ }^{1}$, Yu Lu${ }^{1}$, Xianjun Lao ${ }^{1}$, Zhiping Chen ${ }^{2}$, Ruolin $\mathrm{Li}^{3}$, Jingzhe Sui ${ }^{1}$ Xue Qin ${ }^{1 *}$ and Shan $\mathrm{Li}^{1}$

\begin{abstract}
Background: The base excision repair (BER) pathway removes DNA damage caused by ionizing radiation, reactive oxidative species and methylating agents. OGG1 and APE1 are two important genes in the BER pathway. Many epidemiological studies have evaluated the association between polymorphisms in the two BER genes (OGG1 Ser326Cys and APE1 Asp148Glu) and breast cancer risk. However, the results are inconsistent.

Methods: We searched the electronic databases including PubMed, Embase and Cochrane library for all eligible studies for the period up to February 2014. Data were extracted by two independent authors and pooled odds ratios (ORs) with corresponding 95\% confidence intervals (Cls) were used to assess the strength of the association.

Results: A total of 17 studies including 9,040 cases and 10,042 controls were available for OGG1 Ser326Cys polymorphism and 7 studies containing 2,979 cases and 3,111 controls were included for APE1 Asp148Glu polymorphism. With respect to OGG1 Ser326Cys polymorphism, we did not find a significant association with breast cancer risk when all eligible studies were pooled into the meta-analysis. However, in subgroup analyses by ethnicity and menopausal status, statistical significant increased breast cancer risk was found in Asian populations (Cys/Cys vs. Ser/Ser: OR =1.157, 95\% Cl 1.013-1.321, $P=0.011$; Cys/Cys vs. Ser/Cys + Ser/Ser: OR $=1.113,95 \%$ Cl 1.009-1.227, $P=0.014$ ) and postmenopausal patients (Cys/Cys vs. Ser/Cys + Ser/Ser: OR $=1.162,95 \%$ Cl 1.003-1.346, $P=0.024)$. In subgroup analysis according to quality score, source of control, and HWE in controls, no any significant association was detected. With respect to APE1 Asp148Glu polymorphism, no significant association with breast cancer risk was demonstrated in the overall and stratified analyses.
\end{abstract}

Conclusions: The present meta-analysis suggests that the OGG1 Ser326Cys polymorphism may be a risk factor for breast cancer in Asians and postmenopausal patients. Further large and well-designed studies are needed to confirm this association.

Virtual Slides: The virtual slide(s) for this article can be found here: http://www.diagnosticpathology.diagnomx.eu/ vs/1156934297124915

Keywords: Breast cancer, OGG1, APE1, Polymorphism, Meta-analysis

\footnotetext{
*Correspondence: qinxue919@126.com

'Department of Clinical Laboratory, First Affiliated Hospital of Guangxi

Medical University, Nanning, Guangxi 530021, China

Full list of author information is available at the end of the article
} 


\section{Background}

Breast cancer is currently the most frequently occurring cancer and one of the leading causes of cancer-related death of females worldwide, which has become a major public health challenge $[1,2]$. Breast cancer is a heterogeneous disease such that they may have different prognoses and respond to therapy differently despite similarities in histological types, grade and stage [3,4]. Because of the heterogeneity of breast cancer, the mechanism of breast carcinogenesis is still not fully understood. It has been well established that exposure to various endogenous and exogenous mutagens or carcinogens played a critical role in the development of breast cancer $[5,6]$. The exposures can lead to DNA damage which, if remained unrepaired, may result in genetic instability and unregulated cell growth, and eventually breast cancer [7]. The DNA repairing systems, composed of many DNA repair genes, play a critical role in removing damaged genes resulting from endogenous and exogenous mutagenic exposures, and maintaining the genomic integrity and preventing carcinogenesis.

The base excision repair (BER) pathway is one of the most important DNA repair mechanisms responsible for the repair of DNA damage. It is the most common route for removal of small lesions from DNA and is an important part of cellular defense against a large variety of structurally unrelated DNA lesions. It is believed to be the predominant pathway used for removal of oxidized and many alkylated bases $[8,9]$. BER is initiated by recognition and excision of damaged base by the specific DNA glycosylase. Mammalian cells contain a series of different genes (each with a specialized function), of which 8-oxoguanine glycosylase-1 (OGG1), and apurinic/apyimidinic endonuclease 1 (APE1) genes are two key enzymes in this repair pathway [10]. OGG1 maps on chromosome 3p26.2 and encodes the DNA repair enzyme OGGl responsible for the excision of 8-oxo-7, 8-dihydroguanine (8-oxoG) and other oxidatively damaged DNA bases. The APEX1 gene consists of five exons and four introns spanning $2.21 \mathrm{~kb}$. This gene is located on chromosome 14q11.2-q12. By hydrolyzing 3'-blocking fragments from oxidized DNA, APEX1 produces normal 3'-hydroxyl nucleotide termini that are necessary for DNA repair synthesis and ligation at single- or double-strand breaks [11,12]. Many single nucleotide polymorphisms in the OGG1 and APEX1 gene have been reported, including the commonly occurring Ser326Cys in OGG1 (rs1052133 in dbSNP) and Asp148Glu in APEX1 (rs3136820 in dbSNP). These nonconservative amino acid alterations have been reported to reduce DNA repair activity and consequently increase cancer risk [13,14].

To date, many epidemiological studies have been performed to evaluate the association between OGG1 Ser326Cys and APEX1 Asp148Glu polymorphisms and breast cancer risk, but the results remain conflicting rather than conclusive. With respect to APEX1 Asp148Glu polymorphism, a meta-analysis by Wu et al. [15] found that the APEX1 Asp148Glu polymorphism may not contribute to breast cancer risk, however, they failed to include all eligible studies in the meta-analysis $[16,17]$, which make their conclusions questionable. With respect to OGG1 Ser326Cys polymorphism, two meta-analyses $[18,19]$ investigating the same hypothesis, quite similar in methods and performed almost at the same time, yielded different conclusions. Furthermore, the two previous meta-analyses did not cover all eligible studies [16,17,20-22]. The exact relationship between genetic polymorphisms of OGG1 Ser326Cys and APEX1 Asp148Glu and breast cancer susceptibility has not been entirely established. To provide the most comprehensive assessment of the associations between OGG1 Ser326Cys and APEX1 Asp148Glu polymorphisms and breast cancer risk, we performed an updated meta-analysis of all available studies.

\section{Methods}

\section{Search strategy}

We conducted a comprehensive literature search in PubMed, Embase, and Cochrane library databases for all eligible studies (updated to February 01, 2014) using the following search strategy: ("breast cancer") and ("OGG1", "hOGG1", "APEX1" or "APEX") and ("polymorphism", "variation", "mutation", "genotype", or "genetic polymorphism"). There was no restriction on time period, sample size, population, language, or type of report. All eligible studies were retrieved and their references were checked for other relevant studies. The literature retrieval was performed in duplication by two independent reviewers (Qiliu Peng and Shi Yang). When multiple publications reported on the same or overlapping data, we chose the most recent or largest population. When a study reported the results on different subpopulations, we treated it as separate studies in the meta-analysis.

\section{Selection criteria}

Studies were included if they met the following criteria: (1) Case-control studies which evaluated the association between OGG1 Ser326Cys and APEX1 Asp148Glu polymorphisms and breast cancer risk; (2) had an odds ratio (OR) with 95\% confidence interval (CI) or other available data for estimating OR (95\% CI); and (3) control population did not contain malignant tumor patients. Studies were excluded if one of the following existed: (1) no control population; (2) duplicate of previous publication; and (3) insufficient information for data extraction; (4) Family-based studies of pedigrees with several affected cases per family were also excluded, because their analysis is based on linkage considerations. 


\section{Data extraction}

Two investigators (Qiliu Peng and $\mathrm{Yu} \mathrm{Lu}$ ) independently reviewed and extracted data from all eligible studies. To ensure the accuracy of the information extracted, the two investigators checked the data extraction results and reached consensus on all of the items. If different results were generated, they would check the data again and have a discussion to come to an agreement. If these two authors could not reach a consensus, another author (Xue Qin) was consulted to resolve the dispute and a final decision was made by the majority of the votes. Data extracted from eligible studies included the first author, year of publication, country of origin, ethnicity, genotyping method, matching criteria, source of control, breast cancer confirmation, total numbers of cases and controls and genotype frequencies of cases and controls. When data were otherwise unavailable, we contacted the corresponding author by e-mail for original information. Ethnic backgrounds were categorized as Caucasian, Asian, and Africans. When a study did not state the ethnic descendent or if it was impossible to separate participants according to such phenotype, the group reported was termed as "mixed ethnicity". Menopausal status was divided into premenopausal and postmenopausal and was additionally recorded for the stratified analysis.

\section{Quality score evaluation}

The quality of eligible studies was evaluated independently by two authors (Xue Qin and Qiliu Peng) according to a set of predefined criteria (Additional file 1: Table S1) based on the scale of Thakkinstian et al. [23]. The revised criteria cover the representativeness of cases, source of controls, ascertainment of breast cancer, total sample size, quality control of genotyping methods, and Hardy-Weinberg equilibrium (HWE) in the control population. Disagreements were resolved by consensus. Scores ranged from 0 (lowest) to 10 (highest). Articles with scores equal to or less than 6 were considered "low-quality" studies, whereas those with scores higher than 6 were considered "highquality" studies.

\section{Statistical analysis}

The strength of the association between OGG1 Ser326Cys and APEX1 Asp148Glu polymorphisms and breast cancer risk was assessed by odds ratios (ORs) with $95 \%$ confidence intervals (CIs). The significance of the pooled OR was determined by $\mathrm{Z}$ test and a $p$ value less than 0.05 was considered significant. The association of OGG1 Ser326Cys and APEX1 Asp148Glu polymorphisms with breast cancer risk was assessed using additive models, recessive model, and dominant model. Heterogeneity assumption was checked by a chi-square-based Q-test [24]. A $P_{h}$ value equal to or greater than 0.10 for the Qtest indicates a lack of heterogeneity among studies, and so the fixed-effects model was used for the meta-analysis [25]. Otherwise, the random-effects model was used [26]. Subgroup analyses were performed by ethnicity, menopausal status, quality score, source of control, and HWE in controls. Sensitivity analysis was performed by sequential omission of individual studies. For each polymorphism, publication bias was evaluated using a funnel plot and Egger's regression asymmetry test. The distribution of the genotypes in the control population was tested for HWE using a goodness-of-fit Chi-square test. All analyses were performed using Stata software, version 12.0 (Stata Corp., College Station, TX). All $p$ values were two-sided. To ensure the reliability and the accuracy of the results, two authors entered the data into the statistical software programs independently with the same results.

\section{Results}

\section{Study characteristics}

Based on our search criteria, 24 studies relevant to the role of OGG1 Ser326Cys and APEX1 Asp148Glu polymorphisms on breast cancer susceptibility were identified. Seven of these articles were excluded: one was a review [27], one contained overlapping data [28], two did not present sufficient data for calculating OR and 95\% CI $[29,30]$, and three were meta-analysis $[15,18,19]$. Manual search of references cited in the eligible studies identified 1 additional article [16]. As a result, a total of 18 relevant studies [16,17,20-22,31-43] met the inclusion criteria for the meta-analysis. Among them, two studies $[32,42]$ contained data on two different ethnic groups, and we treated them independently. Therefore, a total of 20 separate comparisons were finally included in the meta-analysis (Figure 1). Of all eligible studies, 13 studies evaluated the OGG1 Ser326Cys polymorphism, 3 studies evaluated the APEX1 Asp148Glu polymorphism, and 4 studies evaluated OGG1 Ser326Cys and APEX1 Asp148Glu polymorphisms simultaneously. Therefore, a total of 17 studies including 9,040 cases and 10,042 controls were available for hOGG1 Ser326Cys polymorphism and 7 studies containing 2,979 cases and 3,111 controls were included for APE1 Asp148Glu polymorphism. Table 1 list all essential information such as the publication year, first author, country, ethnicity, sample size, genotyping methods, source of controls, matching criteria, and breast cancer confirmation for OGG1 Ser326Cys and APEX1 Asp148Glu polymorphisms. The genotype distributions of the controls in 2 studies [32,41] were not consistent with HWE for OGG1 Ser326Cys polymorphism and 1 was not consistent with HWE for APEX1 Asp148Glu polymorphism [17].

\section{Meta-analysis results}

Table 2 lists the main results of meta-analysis of OGG1 Ser326Cys polymorphism and breast cancer risk. The 


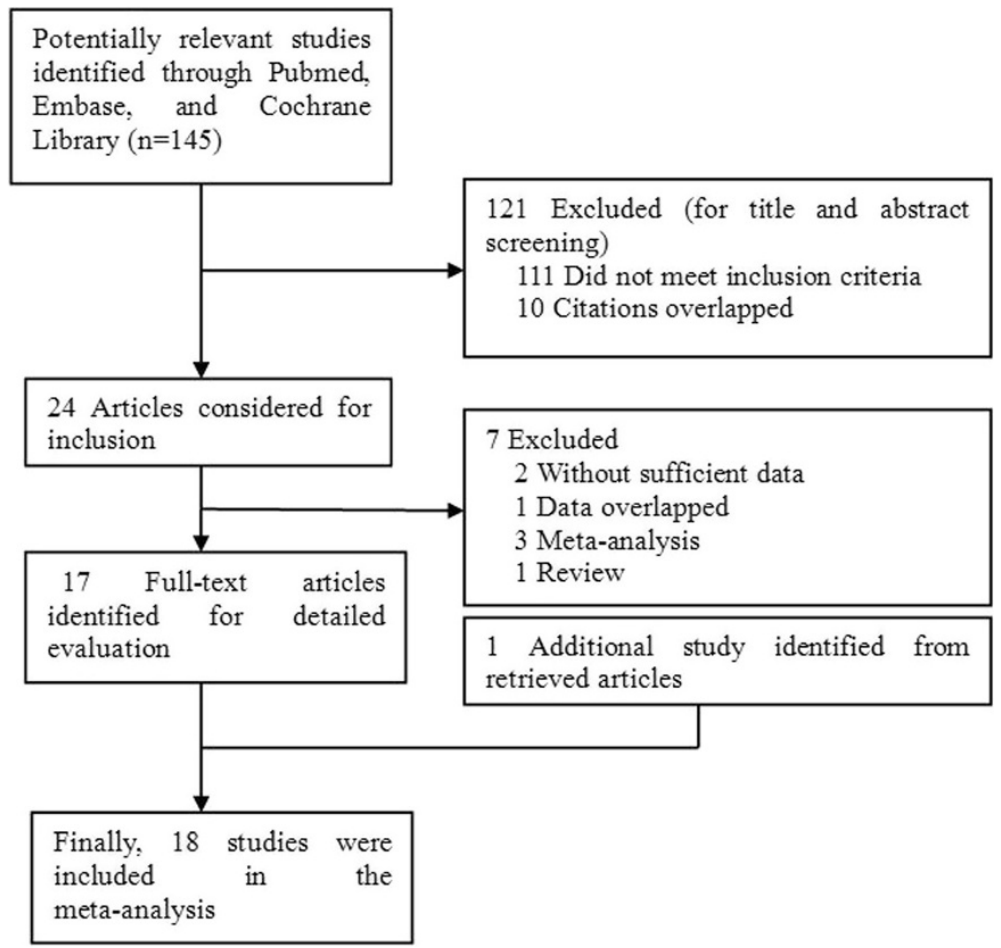

Figure 1 Flowchart of selection of studies for inclusion in meta-analysis.

between-study heterogeneity was not significant in the overall populations and all subgroup analyses (all $P_{h}$ values were larger than 0.1 ), thus the fixed-effects model was used to pool the results. Overall, significant elevated breast cancer risk was not found when all studies were pooled into the meta-analysis. When stratified by quality score, source of control, and HWE in controls, significant increased breast cancer risk was also not detected in all subgroups. However, in subgroup analysis by ethnicity, significant increased breast cancer risk was found in Asians (Cys/Cys vs. Ser/Ser: OR $=1.157,95 \%$ CI 1.0131.321, $P=0.011 ;$ Cys $/$ Cys vs. Ser $/$ Cys + Ser/Ser: OR $=$ 1.113, 95\% CI 1.009-1.227, $P=0.014$; Figure 2) but not in Caucasians. In stratified analysis by menopausal status, significant increased breast cancer risk was observed in postmenopausal patients (Cys/Cys vs. Ser/Cys + Ser/Ser: $\mathrm{OR}=1.162$, 95\% CI 1.003-1.346, $P=0.024$; Figure 3) but not in premenopausal subjects.

Table 3 lists the main results of meta-analysis of APEX1 Asp148Glu polymorphism and breast cancer risk. When all 7 studies were pooled into the meta-analysis, there was no evidence of significant association between APEX1 Asp148Glu polymorphism and breast cancer risk (Glu/Glu vs. Asp/Asp: $\mathrm{OR}=0.948,95 \% \mathrm{CI}=0.817-1.101, P=0.486$; Asp/Glu vs. Asp/Asp: $\mathrm{OR}=0.975,95 \% \mathrm{CI}=0.790-1.204$, $P=0.814 ; \mathrm{Glu} / \mathrm{Glu}+\mathrm{Asp} / \mathrm{Glu}$ vs. Asp/Asp: $\mathrm{OR}=0.959$, 95\% CI $=0.803-1.146, P=0.646$; Glu/Glu vs. Asp/Glu + Asp/Asp: OR $=0.961,95 \% \mathrm{CI}=0.846-1.091, P=0.540)$. In stratified analyses by ethnicity, menopausal status, quality score, source of control, and HWE in controls, statistically significant association was also not found in all subgroups.

\section{Sensitivity analysis}

Sensitivity analysis was performed by sequential omission of individual studies for both OGG1 Ser326Cys and APEX1 Asp148Glu polymorphisms. For analyses of pooling more than three individual studies, the significance of ORs was not influenced excessively by omitting any single study (data not shown). For the OGG1 Ser326Cys polymorphism, sensitivity analysis was further performed by omitting the studies by Choi et al. [32] and Ming-Shiean et al. [41] in which the control populations were not in accordance with HWE. The significance of all ORs was not altered after excluding these two studies. For the APEX1 Asp148Glu polymorphism, a sensitivity analysis was also further performed by omitting the study by Kim et al. [17] in which the control populations were deviated from HWE, and the significance of all ORs was also not altered.

\section{Publication bias}

Begg's funnel plot and Egger's test were performed to access the publication bias in this meta-analysis. The shape of the funnel plot did not reveal any evidence of obvious asymmetry. Then, the Egger's test was used to provide statistical evidence of funnel plot symmetry. The results still did not suggest any evidence of publication bias in 
Table 1 Characteristics of studies included in this meta-analysis

\begin{tabular}{|c|c|c|c|c|c|c|c|c|c|c|}
\hline \multirow{2}{*}{$\begin{array}{l}\text { First author } \\
\text { (Year) }\end{array}$} & \multirow[t]{2}{*}{ Ethnicity } & \multirow{2}{*}{$\begin{array}{l}\text { Sample size } \\
\text { (case/control) }\end{array}$} & \multirow{2}{*}{$\begin{array}{l}\text { Genotyping } \\
\text { methods }\end{array}$} & \multirow[t]{2}{*}{ Matching criteria } & \multirow{2}{*}{$\begin{array}{l}\text { Source of } \\
\text { control }\end{array}$} & \multirow[t]{2}{*}{ BC confirmation } & \multirow{2}{*}{$\begin{array}{l}\text { Quality } \\
\text { scores }\end{array}$} & \multirow[t]{2}{*}{ SNP studied } & \multicolumn{2}{|c|}{ HWE( $P$ value $)$} \\
\hline & & & & & & & & & Ser326Cys & Asp148Glu \\
\hline Vogel [31] & Caucasian & $425 / 434$ & Sequencing & $\begin{array}{l}\text { Age, menopausal } \\
\text { status }\end{array}$ & PB & NA & 7 & OGG1 Ser326Cys & 0.175 & - \\
\hline Choi [32] & Asian & $265 / 284$ & PCR-RFLP & NA & $\mathrm{HB}$ & Histopatho- & 6.5 & OGG1 Ser326Cys & 0.048 & - \\
\hline Choi [32] & Asian & 201/184 & PCR-CTPP & Age & $\mathrm{HB}$ & Histo- & 6 & OGG1 Ser326Cys & 0.914 & - \\
\hline Huang [16] & Asian & $136 / 232$ & PCR-RFLP & Age & $\mathrm{HB}$ & Patho- & 6 & $\begin{array}{l}\text { OGG1 Ser326Cys } \\
\text { APEX1 Asp148Glu }\end{array}$ & 0.525 & 0.565 \\
\hline Zhang [33] & Caucasian & $1571 / 1244$ & TaqMan & Age & PB & NA & 8 & $\begin{array}{l}\text { OGG1 Ser326Cys } \\
\text { APEX1 Asp148Glu }\end{array}$ & 0.930 & 0.456 \\
\hline Rossner [34] & Caucasian & 1041/1093 & FP-TDI & Age & PB & NA & 8 & OGG1 Ser326Cys & 0.857 & - \\
\hline Cai [35] & Asian & $1102 / 1167$ & TaqMan & Age & PB & NA & 8 & OGG1 Ser326Cys & 0.080 & - \\
\hline Synowiec [36] & Caucasian & $41 / 48$ & PCR-RFLP & Age & $\mathrm{HB}$ & NA & 2.5 & OGG1 Ser326Cys & 0.507 & - \\
\hline Sangrajrang [37] & Asian & $506 / 424$ & Capillary PCR & $\begin{array}{l}\text { Region, menopausal } \\
\text { status, drinking, } \\
\text { smoking }\end{array}$ & $\mathrm{HB}$ & Histo- & 7 & $\begin{array}{l}\text { OGG1 Ser326Cys } \\
\text { APEX1 Asp148Glu }\end{array}$ & 0.627 & 0.135 \\
\hline $\begin{array}{l}\text { Romanowicz- } \\
\text { Makowska [38] }\end{array}$ & Caucasian & $100 / 106$ & PCR-RFLP & Age & $\mathrm{HB}$ & NA & 2.5 & OGG1 Ser326Cys & 0.988 & - \\
\hline Loizidou [39] & Caucasian & $1108 / 1174$ & TaqMan & Age & PB & Patho- & 9.5 & OGG1 Ser326Cys & 0.499 & - \\
\hline Sterpone [28] & Caucasian & $43 / 34$ & PCR-SSCP & Age, gender & $\mathrm{HB}$ & NA & 3 & OGG1 Ser326Cys & 0.577 & - \\
\hline Ming-Shiean [41] & Asian & $401 / 533$ & TaqMan & Age & $\mathrm{HB}$ & Patho- & 6.5 & OGG1 Ser326Cys & 0.034 & - \\
\hline Roberts [20] & Caucasian & 1054/1887 & MALDI-TOF & Age, race & PB & Histo- & 8 & OGG1 Ser326Cys & 0.543 & - \\
\hline Smolarz [22] & Caucasian & $70 / 70$ & PCR-RFLP & Age & $\mathrm{HB}$ & Histo- & 5 & OGG1 Ser326Cys & 0.473 & - \\
\hline Kim [17] & Asian & $346 / 351$ & ASPE & $\begin{array}{l}\text { Age, BMl, smoking, } \\
\text { drinking }\end{array}$ & $\mathrm{HB}$ & Histo- & 6.5 & $\begin{array}{l}\text { OGG1 Ser326Cys } \\
\text { APEX1 Asp148Glu }\end{array}$ & 0.296 & 0.012 \\
\hline Xie [21] & Asian & 630/777 & Sequencing & $\begin{array}{l}\text { Age, BMl, } \\
\text { menopausal status }\end{array}$ & $\mathrm{HB}$ & Patho- & 7 & OGG1 Ser326Cys & 0.161 & - \\
\hline Smith [42] & Caucasian & $319 / 405$ & TaqMan & Age, race & $\mathrm{HB}$ & Histopatho- & 7 & APEX1 Asp148Glu & - & 0.507 \\
\hline Smith [42] & African & $53 / 75$ & TaqMan & Age, race & $\mathrm{HB}$ & Histopatho- & 6 & APEX1 Asp148Glu & - & 0.566 \\
\hline Jelonek [43] & Caucasian & $91 / 412$ & PCR-RFLP & Age, gender & PB & NA & 5 & APEX1 Asp148Glu & - & 0.092 \\
\hline
\end{tabular}

BC, breast cancer; Histopatho-, Histopathologically confirmed; Histo-, Histologically confirmed; Patho-, Pathologically confirmed; NA, Not available; PB, Population-based; HB, Hospital-based; HWE, Hardy-Weinberg

primer extension and detection by fluorescence polarization; PCR-SSCP, Polymerase chain reaction-single strand conformation polymorphism; MALDI-TOF, Matrix-assisted laser desorption/ionization-time of flight mass

spectrometry; ASPE, Allele-specific primer extension method. 


\begin{tabular}{|c|c|c|c|c|c|c|c|c|c|}
\hline \multirow[t]{2}{*}{ Analysis } & \multirow[t]{2}{*}{$\begin{array}{l}\text { No. of } \\
\text { studies }\end{array}$} & \multicolumn{2}{|c|}{$\begin{array}{l}\text { Cys/Cys vs. Ser/Ser } \\
\text { (Homozygote) }\end{array}$} & \multicolumn{2}{|l|}{$\begin{array}{l}\text { Ser/Cys vs. Ser/Ser } \\
\text { (Heterozygote) }\end{array}$} & \multicolumn{2}{|c|}{$\begin{array}{l}\text { Cys/Cys + Ser/Cys vs. Ser/Ser } \\
\text { (Dominant model) }\end{array}$} & \multicolumn{2}{|c|}{$\begin{array}{l}\text { Cys/Cys vs. Ser/Cys + Ser/Ser } \\
\text { (Recessive model) }\end{array}$} \\
\hline & & OR $(95 \% \mathrm{Cl})$ & $P / P_{\mathrm{h}}$ & OR $(95 \% \mathrm{Cl})$ & $P / P_{\mathrm{h}}$ & OR $(95 \% \mathrm{Cl})$ & $P / P_{\mathrm{h}}$ & OR $(95 \% \mathrm{Cl})$ & $P / P_{\mathrm{h}}$ \\
\hline Overall & 17 & $1.073(0.968-1.190)$ & $0.180 / 0.485$ & $0.986(0.923-1.054)$ & $0.679 / 0.594$ & $1.001(0.940-1.067)$ & $0.971 / 0.501$ & $1.079(0.993-1.172)$ & $0.074 / 0.580$ \\
\hline \multicolumn{10}{|l|}{ Ethnicity } \\
\hline Caucasian & 9 & $0.956(0.812-1.127)$ & $0.595 / 0.635$ & $0.964(0.891-1.043)$ & $0.357 / 0.218$ & $0.967(0.897-1.043)$ & $0.388 / 0.271$ & $0.995(0.850-1.164)$ & $0.945 / 0.958$ \\
\hline Asian & 8 & $1.157(1.013-1.321)$ & $0.011 / 0.492$ & $1.043(0.922-1.178)$ & $0.505 / 0.946$ & $1.085(0.967-1.218)$ & $0.166 / 0.903$ & $1.113(1.009-1.227)$ & $0.014 / 0.173$ \\
\hline \multicolumn{10}{|l|}{ Menopausal status } \\
\hline Premenopausal & 7 & $1.153(0.957-1.388)$ & $0.134 / 0.215$ & $1.030(0.897-1.182)$ & $0.677 / 0.995$ & $1.052(0.923-1.199)$ & $0.449 / 0.855$ & $1.175(0.929-1.487)$ & $0.178 / 0.149$ \\
\hline Postmenopausal & 9 & $1.154(0.965-1.380)$ & $0.116 / 0.710$ & $0.962(0.863-1.072)$ & $0.482 / 0.202$ & $0.994(0.896-1.102)$ & $0.903 / 0.351$ & $1.162(1.003-1.346)$ & $0.024 / 0.570$ \\
\hline \multicolumn{10}{|l|}{ Quality score } \\
\hline$>6$ & 11 & $1.093(0.980-1.218)$ & $0.110 / 0.845$ & $0.993(0.928-1.063)$ & $0.841 / 0.941$ & $1.007(0.944-1.075)$ & $0.829 / 0.912$ & $1.086(0.995-1.186)$ & $0.065 / 0.507$ \\
\hline$\leq 6$ & 6 & $0.916(0.664-1.262)$ & $0.590 / 0.110$ & $0.879(0.667-1.159)$ & $0.361 / 0.157$ & $0.910(0.702-1.180)$ & 0.478/0.170 & $1.017(0.789-1.310)$ & $0.899 / 0.446$ \\
\hline \multicolumn{10}{|l|}{ Source of control } \\
\hline$H B$ & 11 & $1.134(0.975-1.320)$ & $0.104 / 0.164$ & $0.966(0.842-1.107)$ & $0.615 / 0.384$ & $1.029(0.904-1.170)$ & $0.669 / 0.238$ & $1.166(0.945-1.307)$ & $0.107 / 0.462$ \\
\hline PB & 6 & $1.023(0.889-1.178)$ & 0.750/0.997 & $0.992(0.920-1.071)$ & $0.845 / 0.656$ & $0.993(0.923-1.067)$ & $0.843 / 0.802$ & $0.987(0.874-1.114)$ & 0.829/0.987 \\
\hline \multicolumn{10}{|l|}{ HWE in controls } \\
\hline Yes & 15 & $1.071(0.960-1.195)$ & $0.218 / 0.343$ & $0.989(0.924-1.059)$ & $0.757 / 0.468$ & $1.002(0.939-1.069)$ & $0.961 / 0.362$ & $1.068(0.976-1.168)$ & $0.465 / 0.154$ \\
\hline No & 2 & $1.086(0.803-1.471)$ & $0.592 / 0.993$ & $0.929(0.693-1.244)$ & $0.620 / 0.717$ & $0.993(0.755-1.307)$ & $0.961 / 0.765$ & $1.142(0.923-1.413)$ & $0.223 / 0.726$ \\
\hline
\end{tabular}

$P_{\mathrm{h}} \mathrm{P}$ values of Q-test for heterogeneity test. OR, odds ratio; $\mathrm{Cl}$, confidence intervals; $\mathrm{HB}$, Hospital-based studies; $\mathrm{PB}$, Population-based studies; $\mathrm{HWE}$, Hardy-Weinberg equilibrium. 


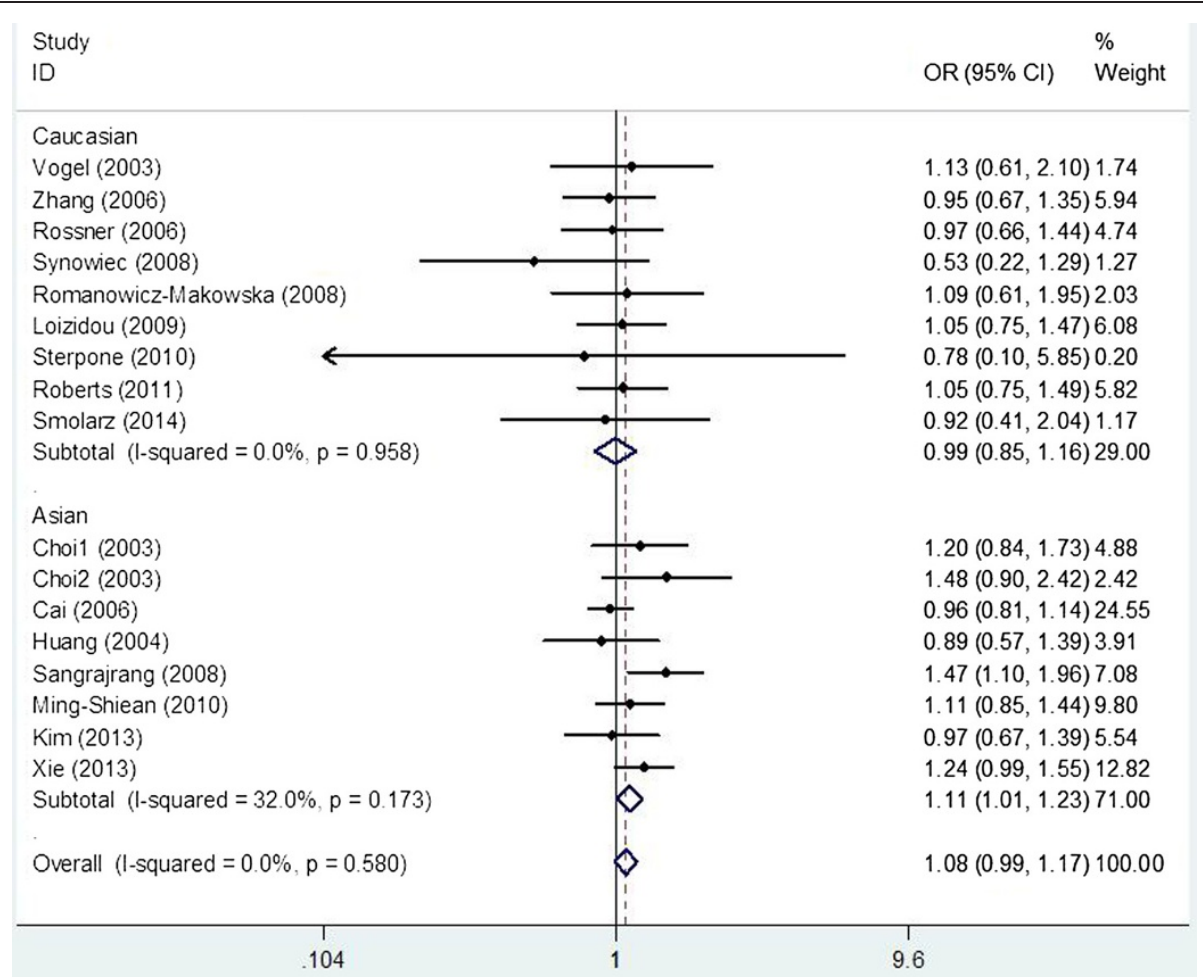

Figure 2 Forest plots of OGG1 Ser326Cys polymorphism and breast cancer risk in subgroup analysis by ethnicity using a fixed-effect model (Recessive model Cys/Cys vs. Ser/Cys + Ser/Ser).

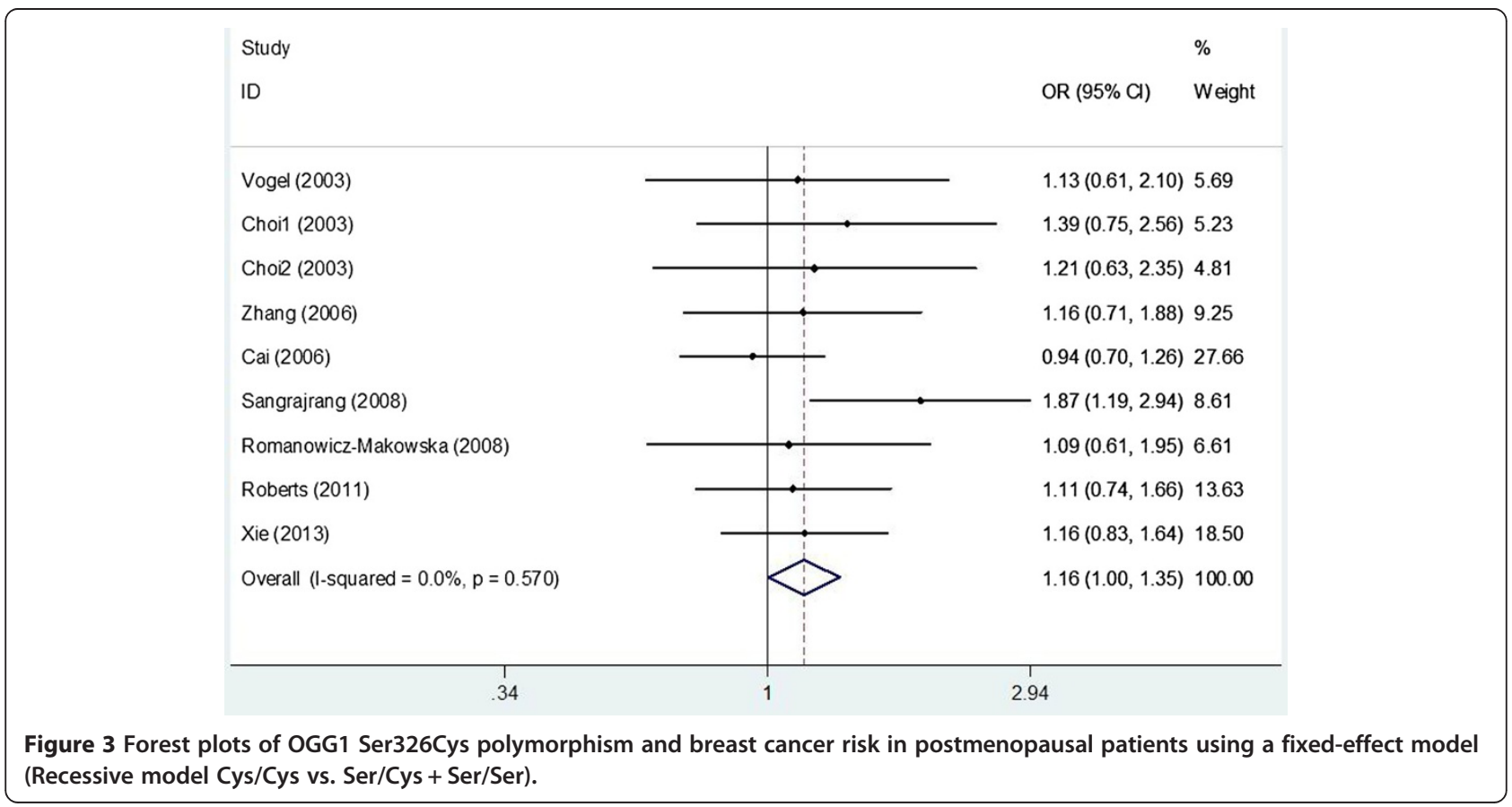


Table 3 Meta-analysis of APEX1 Asp148Glu polymorphism and breast cancer risk

\begin{tabular}{|c|c|c|c|c|c|c|c|c|c|}
\hline \multirow[t]{2}{*}{ Analysis } & \multirow[t]{2}{*}{$\begin{array}{l}\text { No. of } \\
\text { studies }\end{array}$} & \multicolumn{2}{|c|}{ Glu/Glu vs. Asp/Asp (Homozygote) } & \multicolumn{2}{|c|}{ Asp/Glu vs. Asp/Asp (Heterozygote) } & \multicolumn{2}{|c|}{$\begin{array}{l}\text { Glu/Glu + Asp/Glu vs. Asp/Asp } \\
\text { (Dominant model) }\end{array}$} & \multicolumn{2}{|c|}{$\begin{array}{l}\text { Glu/Glu vs. Asp/Glu + Asp/Asp } \\
\text { (Recessive model) }\end{array}$} \\
\hline & & OR $(95 \% \mathrm{Cl})$ & $P / P_{\mathrm{h}}$ & OR $(95 \% \mathrm{Cl})$ & $P / P_{\mathrm{h}}$ & OR $(95 \% \mathrm{Cl})$ & $P / P_{\mathrm{h}}$ & OR $(95 \% \mathrm{Cl})$ & $P / P_{\mathrm{h}}$ \\
\hline Overall & 7 & $0.948(0.817-1.101)$ & $0.486 / 0.508$ & $0.975(0.790-1.204)$ & $0.814 / 0.028$ & $0.959(0.803-1.146)$ & $0.646 / 0.074$ & $0.961(0.846-1.091)$ & $0.540 / 0.572$ \\
\hline \multicolumn{10}{|l|}{ Ethnicity } \\
\hline Caucasian & 3 & $1.019(0.850-1.221)$ & $0.842 / 0.395$ & $0.928(0.670-1.285)$ & $0.651 / 0.071$ & $0.955(0.717-1.271)$ & $0.751 / 0.097$ & $1.042(0.897-1.211)$ & $0.590 / 0.839$ \\
\hline Asian & 3 & $0.813(0.620-1.066)$ & $0.135 / 0.455$ & $1.031(0.681-1.560)$ & $0.886 / 0.017$ & $0.968(0.680-1.378)$ & $0.855 / 0.035$ & $0.873(0.604-1.091)$ & $0.142 / 0.847$ \\
\hline African & 1 & $0.948(0.817-1.101)$ & $0.794 /-$ & $0.870(0.404-1.870)$ & $0.720 /-$ & $0.870(0.426-1.774)$ & $0.701 /-$ & $0.933(0.353-2.469)$ & $0.889 /-$ \\
\hline \multicolumn{10}{|l|}{ Menopausal status } \\
\hline Premenopausal & 2 & $0.895(0.662-1.209)$ & $0.469 / 0.509$ & $0.938(0.744-1.181)$ & $0.584 / 0.688$ & $0.921(0.741-1.145)$ & $0.460 / 0.602$ & $0.927(0.716-1.201)$ & $0.567 / 0.556$ \\
\hline Postmenopausal & 2 & $0.882(0.515-1.510)$ & 0.648/0.096 & $1.046(0.847-1.290)$ & $0.678 / 0.644$ & $1.021(0.839-1.243)$ & $0.835 / 0.323$ & $0.972(0.782-1.208)$ & $0.797 / 0.113$ \\
\hline \multicolumn{10}{|l|}{ Quality score } \\
\hline$>6$ & 4 & $0.946(0.806-1.109)$ & $0.491 / 0.351$ & $0.999(0.760-1.313)$ & $0.994 / 0.008$ & $0.973(0.779-1.215)$ & $0.807 / 0.031$ & $0.952(0.831-1.091)$ & $0.479 / 0.266$ \\
\hline$\leq 6$ & 3 & $0.968(0.637-1.472)$ & 0.880/0.368 & $0.898(0.642-1.256)$ & $0.528 / 0.389$ & $0.907(0.661-1.245)$ & $0.547 / 0.303$ & $1.024(0.719-1.456)$ & $0.897 / 0.716$ \\
\hline \multicolumn{10}{|l|}{ Source of control } \\
\hline $\mathrm{HB}$ & 5 & $0.821(0.659-1.025)$ & $0.081 / 0.809$ & $0.921(0.669-1.268)$ & $0.612 / 0.011$ & $0.898(0.697-1.157)$ & $0.405 / 0.055$ & $0.864(0.709-1.052)$ & $0.146 / 0.642$ \\
\hline PB & 2 & $1.070(0.874-1.309)$ & $0.513 / 0.398$ & $1.049(0.882-1.248)$ & $0.589 / 0.538$ & $1.055(0.896-1.242)$ & $0.521 / 0.450$ & $1.037(0.878-1.226)$ & $0.667 / 0.562$ \\
\hline \multicolumn{10}{|l|}{ HWE in controls } \\
\hline Yes & 6 & $0.941(0.803-1.104)$ & $0.456 / 0.390$ & $0.930(0.818-1.057)$ & $0.268 / 0.264$ & $0.929(0.824-1.048)$ & $0.232 / 0.258$ & $0.989(0.864-1.132)$ & $0.869 / 0.650$ \\
\hline No & 1 & $1.001(0.654-1.531)$ & $0.997 /-$ & $1.345(0.904-2.162)$ & $0.211 /-$ & $1.362(0.994-1.868)$ & $0.055 /-$ & $0.774(0.532-1.126)$ & $0.180 /-$ \\
\hline
\end{tabular}

$P_{\mathrm{h}} \mathrm{P}$ values of Q-test for heterogeneity test. $\mathrm{OR}$, odds ratio; $\mathrm{Cl}$, confidence intervals; $\mathrm{HB}$, Hospital-based studies; $\mathrm{PB}$, Population-based studies; $\mathrm{HWE}$, Hardy-Weinberg equilibrium. 
OGG1 Ser326Cys $(P=0.104$ for Cys $/$ Cys vs. Ser/Ser; $\mathrm{P}=0.187$ for Ser/Cys vs. Ser/Ser; $\mathrm{P}=0.560$ for recessive model Cys/Cys vs. Ser/Cys + Ser/Ser, Figure 4A; and $\mathrm{P}=0.339$ for dominant model Cys/Cys + Ser/Cys vs. Ser/ Ser) and APEX1 Asp148Glu ( $\mathrm{P}=0.535$ for $\mathrm{Glu} / \mathrm{Glu}$ vs. Asp/Asp; $\mathrm{P}=0.789$ for Asp/Glu vs. Asp/Asp; $\mathrm{P}=0.504$ for recessive model Glu/Glu vs. Asp/Glu + Asp/Asp, Figure 4B; and $\mathrm{P}=0.766$ for dominant model Glu/Glu + Asp/Glu vs. Asp/Asp) polymorphisms.

\section{Discussion}

Maintenance of genomic integrity by DNA repair genes is an essential component of normal cell homeostasis necessary to cell growth, differentiation, and apoptosis $[7,44]$. Previous evidence indicated that reduced DNA repair capacity, due to various DNA repair gene polymorphisms, is associated with increased risk and susceptibility to cancers $[45,46]$. BER pathway is one of the most important DNA repair mechanisms responsible for the repair of DNA damage. It was initiated by recognition and excision of damaged base by the specific DNA glycosylase. OGG1 and APEX1 are both central players in the BER pathway. Many epidemiological studies have been performed to evaluate the role of OGG1 Ser326Cys and APEX1 Asp148Glu polymorphisms on breast cancer risk; however, the results remained conflicting and contradictory. The conflicting results are possibly because of the small effect of OGG1 Ser326Cys and APEX1 Asp148Glu polymorphisms on cancer risk or the relatively low statistical power of individual published studies. To provide the most comprehensive assessment of the association between OGG1 Ser326Cys and APEX1 Asp148Glu polymorphisms and breast cancer risk, we performed this meta-analysis of all available studies. Our results suggested that the OGG1 Ser326Cys polymorphism was significantly associated with increased breast cancer risk in Asian populations and postmenopausal patients. However, our data did not support a significant association between APEX1 Asp148Glu polymorphism and breast cancer risk both in the pooled analysis and stratified analyses.

This finding may be biologically plausible. Oxidative DNA damage induced by reactive oxygen species (ROS) is involved in the process of carcinogenesis. Reactive oxygen species could be generated from estrogen metabolism through catechol estrogen redox cycling $[47,48]$. If not quenched, these reactive oxygen species may cause oxidative DNA damage and increase breast cancer risk. It has been suggested that 8-hydroxyguanine, a major product of oxidative DNA damage, plays an important role in carcinogenesis given its abundant and highly mutagenic properties [49]. 8-Hydroxyguanine is subjected to BER, especially via the 8-oxoguanine DNA glycosylase (OGG1) catalyzing the release of 8-hydroxy-2'-deoxyguanosine and the cleavage of DNA at the AP site. Variants in the OGG1 gene might alter protein structure or function or create alternatively spliced proteins which may influence BER efficiency and hence affect individual susceptibility to cancers. It was reported that the OGG1 326Cys variant enzyme have lower activity than the 326Cys enzyme [50]. More importantly, the homozygous variant genotype OGG1 326Cys has been shown associated with increased risk for many different types of cancers, including colorectal cancer [51], hepatocellular carcinoma [52] and lung cancer [53].

In the stratification analysis of ethnicity, we found an evidence for the association between the hOGG1 Ser326Cys polymorphism and breast cancer susceptibility among Asians but not Caucasians. A possible reason for the
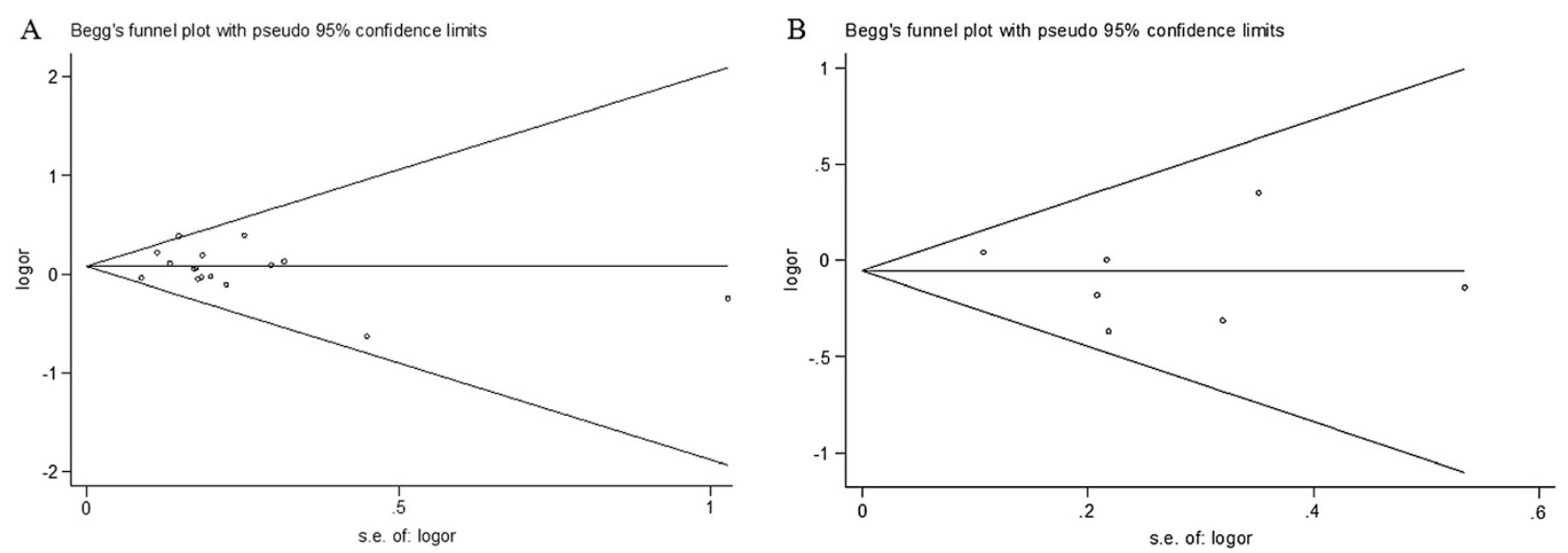

Figure 4 Funnel plot analysis to detect publication bias. Each point represents a separate study for the indicated association. A Funnel plot for recessive model Cys/Cys vs. Ser/Cys + Ser/Ser of OGG1 Ser326Cys polymorphism in the overall analysis $(P=0.560)$; B Funnel plot for recessive model Glu/Glu vs. Asp/Glu + Asp/Asp of APEX1 Asp148Glu polymorphism in overall analysis $(P=0.504)$. 
differences might be the different genetic backgrounds and gene-environment interactions. We observed a wide variation of the Cys allele frequencies of control resources in Asian (0.52) and Caucasian (0.24) population that were very close to that obtained from the HapMap Project ( 0.50 for CHB and 0.22 for CEU), and this different allele frequency might account for the discrepancies between the hOGG1 Ser326Cys polymorphism and breast cancer susceptibility among different ethnicity.

Previous evidence suggested that the menopausal status was one of the important risk factors for the development of breast cancer. So we carried out subgroup analysis according to menopausal status in this meta-analysis. Our results suggested a significant increased breast cancer risk in postmenopausal patients (Cys/Cys vs. Ser/Cys + Ser/ Ser: $\mathrm{OR}=1.162,95 \%$ CI 1.003-1.346, $P=0.024$ ) but not in premenopausal subjects. Though results from available studies investigating OGG1 Ser326Cys polymorphism and breast cancer risk have been inconsistent, our results are consistent with the results of the large sample study by Sangrajrang et al. [37]. The most possible reason for this discrepancy in premenopausal and postmenopausal patients is that the OGG1 Ser326Cys polymorphism plays different roles in repairing oxidative DNA damage resulting from estrogen metabolism in patients with different menopausal status.

Some limitations of this meta-analysis should be addressed. First, in subgroup analysis by ethnicity, the included studies regarded only Asians and Caucasians for OGG1 Ser326Cys polymorphism. Data concerning other ethnicities such as Africans were not found. Thus, additional studies are warranted to evaluate the effect of this functional polymorphism on breast cancer risk in different ethnicities, especially in Africans. Second, our results were based on unadjusted estimates. We did not perform the analysis adjusted for other covariates such as age, obesity, drinking and smoking status, use of contraceptives, environment factors, and so on, because of the unavailable original data of the eligible studies.

\section{Conclusions}

The meta-analysis provided a more precise estimation based on larger sample size compared with the individual studies and previous meta-analysis. Our study suggested that OGG1 Ser326Cys polymorphism might contribute to breast cancer risk, especially in Asian populations and postmenopausal patients. In order to further verify our findings, large well designed epidemiological studies are warranted.

\section{Additional file}

Additional file 1: Table S1. Scale for Quality Assessment.

\section{Abbreviations}

HWE: Hardy-Weinberg equilibrium; BER: Base excision repair; OGG1: Human 8-oxogunaine glycosylase-1; APEX1: Apurinic/apyrimidinic-endonuclease-1; SNP: Single nucleotide polymorphism; OR: Odds ratio; Cl: Confidence interval.

\section{Competing interest}

The authors declare that they have no competing interest.

\section{Authors' contributions}

$\mathrm{QP}, \mathrm{XQ}, \mathrm{YL}, \mathrm{XL}$ performed the literature search, data extraction, statistical analysis and drafted the manuscript. QP, XL, JS, and RL participated in data extraction. QP, SL, XQ, ZC supervised the literature search, data extraction, statistical analysis and drafted the manuscript. All authors read and approved the final manuscript.

\section{Acknowledgments}

The work described in this paper was supported by the National Natural Science Fundation (No. 81260302), Guangxi education and innovation projects for graduate student in 2014 (No. YCBZ2014031).

\section{Author details}

'Department of Clinical Laboratory, First Affiliated Hospital of Guangxi Medical University, Nanning, Guangxi 530021, China. ${ }^{2}$ Department of Occupational Health and Environmental Health, School of Public Health at Guangxi Medical University, Nanning, Guangxi, China. ${ }^{3}$ Department of Medicine Research, First Affiliated Hospital of Guangxi Medical University, Nanning, Guangxi, China.

Received: 23 March 2014 Accepted: 21 May 2014

Published: 3 June 2014

\section{References}

1. Wang S, Li H, Wang J, Wang D: Expression of microRNA-497 and its prognostic significance in human breast cancer. Diagn Pathol 2013, 8:172.

2. Cheng H, Qin Y, Fan H, Su P, Zhang X, Zhang H, Zhou G: Overexpression of CARM1 in breast cancer is correlated with poorly characterized clinicopathologic parameters and molecular subtypes. Diagn Pathol 2013, 8:129.

3. El Fatemi H, Chahbouni S, Jayi S, Moumna K, Melhouf MA, Bannani A, Mesbahi O, Amarti A: Luminal B tumors are the most frequent molecular subtype in breast cancer of North African women: an immunohistochemical profile study from Morocco. Diagn Pathol 2012, 7:170.

4. Oger M, Allaoui M, Elie N, Marnay J, Herlin P, Plancoulaine B, Chasle J, Becette V, Bor-Angelier C: Impact of tumor heterogeneity on disease-free survival in a series of 368 patients treated for a breast cancer. Diagn Pathol 2013, 8:S43.

5. Wakeford R: The cancer epidemiology of radiation. Oncogene 2004, 23:6404-6428.

6. Ronckers CM, Erdmann CA, Land CE: Radiation and breast cancer: a review of current evidence. Breast Cancer Res 2005, 7:21-32.

7. Hoeijmakers JH: Genome maintenance mechanisms for preventing cancer. Nature 2001, 411:366-374.

8. Marnett L: Oxyradicals and DNA damage. Carcinogenesis 2000, 21:361-370.

9. Barzilai A, Yamamoto K: DNA damage responses to oxidative stress. DNA Repair (Amst) 2004, 3:1109-1115.

10. Hung RJ, Hall J, Brennan P, Boffetta P: Genetic polymorphisms in the base excision repair pathway and cancer risk: a HuGE review. Am J Epidemiol 2005, 162:925-942.

11. Izumi T, Hazra TK, Boldogh I, Tomkinson AE, Park MS, Ikeda S, Mitra S: Requirement for human AP endonuclease 1 for repair of 3'-blocking damage at DNA single-strand breaks induced by reactive oxygen species. Carcinogenesis 2000, 21:1329-1334.

12. Kelley MR, Cheng L, Foster R, Tritt R, Jiang J, Broshears J, Koch M: Elevated and altered expression of the multifunctional DNA base excision repair and redox enzyme Ape1/ref-1 in prostate cancer. Clin Cancer Res 2001, 7:824-830.

13. Park J, Chen L, Tockman MS, Elahi A, Lazarus P: The human 8-oxoguanine DNA N-glycosylase 1 (hOGG1) DNA repair enzyme and its association with lung cancer risk. Pharmacogenetics 2004, 14:103-109. 
14. Lo YL, Jou YS, Hsiao CF, Chang GC, Tsai YH, Su WC, Chen KY, Chen YM, Huang MS, Hu CY, Chen CJ, Hsiung CA: A polymorphism in the APE1 gene promoter is associated with lung cancer risk. Cancer Epidemiol Biomarkers Prev 2009, 18:223-229.

15. Wu B, Liu HL, Zhang S, Dong XR, Wu G: Lack of an association between two BER gene polymorphisms and breast cancer risk: a meta-analysis. PLoS One 2012, 7:e50857.

16. Huang CS, Chen JJ, Yang SY, Cheng CW, Chern HD, Chang KJ, Wu PE, Shen CY: Breast Cancer Risk Associated with Genotypic Polymorphism of Oxidative DNA Damage Repair Genes - A Multigenic Study of Base Excision Repair and Transcription-Coupled Repair in Cancer Susceptibility. J Genet Mole Biol 2004, 15:116-136.

17. Kim KY, Han W, Noh DY, Kang D, Kwack K: Impact of genetic polymorphisms in base excision repair genes on the risk of breast cancer in a Korean population. Gene 2013, 532:192-196.

18. Gu D, Wang M, Zhang Z, Chen J: Lack of association between the hOGG1 Ser326Cys polymorphism and breast cancer risk: evidence from 11 case-control studies. Breast Cancer Res Treat 2010, 122:527-531.

19. Yuan W, Xu L, Feng Y, Yang Y, Chen W, Wang J, Pang D, Li D: The hOGG1 Ser326Cys polymorphism and breast cancer risk: a meta-analysis. Breast Cancer Res Treat 2010, 122:835-842.

20. Roberts MR, Shields PG, Ambrosone CB, Nie J, Marian C, Krishnan SS, Goerlitz DS, Modali R, Seddon M, Lehman T, Amend KL, Trevisan M, Edge $S B$, Freudenheim JL: Single-nucleotide polymorphisms in DNA repair genes and association with breast cancer risk in the web study. Carcinogenesis 2011, 32:1223-1230.

21. Xie $H$, Xia K, Rong $H$, Chen X: Genetic polymorphism in hOGG1 is associated with triple-negative breast cancer risk in Chinese Han women. Breast 2013, 22:707-712.

22. Smolarz B, Makowska M, Samulak D, Michalska MM, Mojs E, Wilczak M, Romanowicz $\mathrm{H}$ : Single nucleotide polymorphisms (SNPs) of ERCC2, hOGG1, and XRCC1 DNA repair genes and the risk of triple-negative breast cancer in Polish women. Tumour Biol 2014, 35:3495-3502.

23. Thakkinstian A, McEvoy M, Minelli C, Gibson P, Hancox B, Duffy D, Thompson J, Hall I, Kaufman J, Leung TF, Helms PJ, Hakonarson H, Halpi E, Navon R, Attia J: Systematic review and meta-analysis of the association between \{beta\}2-adrenoceptor polymorphisms and asthma: a HuGE review. Am J Epidemiol 2005, 162:201-211.

24. Higgins JP, Thompson SG: Quantifying heterogeneity in a meta-analysis. Stat Med 2002, 21:1539-1558.

25. Mantel N, Haenszel W: Statistical aspects of the analysis of data from retrospective studies of disease. J Natl Cancer Inst 1959, 22:719-748.

26. DerSimonian R, Laird N: Meta-analysis in clinical trials. Control Clin Trials 1986, 7:177-188

27. Goode EL, Ulrich CM, Potter JD: Polymorphisms in DNA repair genes and associations with cancer risk. Cancer Epidemiol Biomarkers Prev 2002, 11:1513-1530.

28. Sterpone S, Mastellone V, Padua L, Novelli F, Patrono C, Cornetta T, Giammarino D, Donato V, Testa A, Cozzi R: Single-nucleotide polymorphisms in BER and HRR genes, XRCC1 haplotypes and breast cancer risk in Caucasian women. J Cancer Res Clin Oncol 2010, 136:631-636.

29. Sapkota Y, Mackey JR, Lai R, Franco-Villalobos C, Lupichuk S, Robson PJ, Kopciuk K, Cass CE, Yasui Y, Damaraju S: Assessing SNP-SNP interactions among DNA repair, modification and metabolism related pathway genes in breast cancer susceptibility. PLoS One 2013, 8:e64896.

30. Ali MF, Meza JL, Rogan EG, Chakravarti D: Prevalence of BER gene polymorphisms in sporadic breast cancer. Oncol Rep 2008, 19:1033-1038.

31. Vogel U, Nexo BA, Olsen A, Thomsen B, Jacobsen NR, Wallin H, Overvad K, Tjonneland A: No association between OGG1 Ser326Cys polymorphism and breast cancer risk. Cancer Epidemiol Biomarkers Prev 2003, 12:170-171.

32. Choi JY, Hamajima N, Tajima K, Yoo KY, Yoon KS, Park SK, Kim SU, Lee KM, Noh DY, Ahn SH, Choe K, Han W, Hirvonen A, Kang D: hOGG1 Ser326Cys polymorphism and breast cancer risk among Asian women. Breast Cancer Res Treat 2003, 79:59-62.

33. Zhang Y, Newcomb PA, Egan KM, Titus-Ernstoff L, Chanock S, Welch R, Brinton LA, Lissowska J, Bardin-Mikolajczak A, Peplonska B, Szeszenia-Dabrowska N, Zatonski W, Garcia-Closas M: Genetic polymorphisms in base-excision repair pathway genes and risk of breast cancer. Cancer Epidemiol Biomarkers Prev 2006, 15:353-358.

34. Rossner P Jr, Terry MB, Gammon MD, Zhang FF, Teitelbaum SL, Eng SM, Sagiv SK, Gaudet MM, Neugut Al, Santella RM: OGG1 polymorphisms and breast cancer risk. Cancer Epidemiol Biomarkers Prev 2006, 15:811-815.
35. Cai Q, Shu XO, Wen W, Courtney R, Dai Q, Gao YT, Zheng W: Functional Ser326Cys polymorphism in the hOGG1 gene is not associated with breast cancer risk. Cancer Epidemiol Biomarkers Prev 2006, 15:403-404

36. Synowiec E, Stefanska J, Morawiec Z, Blasiak J, Wozniak K: Association between DNA damage, DNA repair genes variability and clinical characteristics in breast cancer patients. Mutat Res 2008, 648:65-72.

37. Sangrajrang $S$, Schmezer $P$, Burkholder I, Waas $P$, Boffetta $P$, Brennan $P$, Bartsch H, Wiangnon S, Popanda O: Polymorphisms in three base excision repair genes and breast cancer risk in Thai women. Breast Cancer Res Treat 2008, 111:279-288.

38. Romanowicz-Makowska H, Smolarz B, Makowski M, Polac I, Pertynski T: Ser326Cys polymorphism in DNA repair genes hOGG1 in breast cancer women. Pol J Pathol 2008, 59:201-204.

39. Loizidou MA, Michael T, Neuhausen SL, Newbold RF, Marcou Y, Kakouri E, Daniel M, Papadopoulos P, Malas S, Hadjisavvas A, Kyriacou K: DNA-repair genetic polymorphisms and risk of breast cancer in Cyprus. Breast Cancer Res Treat 2009, 115:623-627.

40. Sterpone S, Cornetta T, Padua L, Mastellone V, Giammarino D, Testa A, Tirindelli D, Cozzi R, Donato V: DNA repair capacity and acute radiotherapy adverse effects in Italian breast cancer patients. Mutat Res 2010, 684:43-48.

41. Ming-Shiean H, Yu JC, Wang HW, Chen ST, Hsiung CN, Ding SL, Wu PE, Shen CY, Cheng CW: Synergistic effects of polymorphisms in DNA repair genes and endogenous estrogen exposure on female breast cancer risk. Ann Surg Oncol 2010, 17:760-771.

42. Smith TR, Levine EA, Freimanis Rl, Akman SA, Allen GO, Hoang KN, Liu-Mares W, $\mathrm{Hu} J \mathrm{~J}$ : Polygenic model of DNA repair genetic polymorphisms in human breast cancer risk. Carcinogenesis 2008, 29:2132-2138.

43. Jelonek K, Gdowicz-Klosok A, Pietrowska M, Borkowska M, Korfanty J, Rzeszowska-Wolny J, Widlak P: Association between single-nucleotide polymorphisms of selected genes involved in the response to DNA damage and risk of colon, head and neck, and breast cancers in a Polish population. J Appl Genet 2010, 51:343-352.

44. Wood RD, Mitchell M, Sgouros J, Lindahl T: Human DNA repair genes. Science 2001, 291:1284-1289.

45. Zhang $L$, Zhang Z, Yan W: Single nucleotide polymorphisms for DNA repair genes in breast cancer patients. Clin Chim Acta 2005, 359:150-155.

46. Garcia-Closas M, Malats N, Real FX, Welch R, Kogevinas M, Chatterjee N, Pfeiffer R, Silverman D, Dosemeci M, Tardon A, Serra C, Carrato A, Garcia-Closas R, Castano-Vinyals G, Chanock S, Yeager M, Rothman N: Genetic variation in the nucleotide excision repair pathway and bladder cancer risk. Cancer Epidemiol Biomarkers Prev 2006, 15:536-542

47. Roy D, Liehr JG: Estrogen, DNA damage and mutations. Mutat Res 1999, 424:107-115.

48. Yoshie $\mathrm{Y}$, Ohshima $\mathrm{H}$ : Synergistic induction of DNA strand breakage by catechol-estrogen and nitric oxide: implications for hormonal carcinogenesis. Free Radic Biol Med 1998, 24:341-348.

49. Floyd RA: The role of 8-hydroxyguanine in carcinogenesis. Carcinogenesis 1990, 11:1447-1450.

50. Kohno T, Shinmura K, Tosaka M, Tani M, Kim SR, Sugimura H, Nohmi T, Kasai H, Yokota J: Genetic polymorphisms and alternative splicing of the hOGG1 gene, that is involved in the repair of 8-hydroxyguanine in damaged DNA. Oncogene 1998, 16:3219-3225.

51. Canbay E, Cakmakoglu B, Zeybek U, Sozen S, Cacina C, Gulluoglu M, Balik E, Bulut T, Yamaner S, Bugra D: Association of APE1 and hOGG1 polymorphisms with colorectal cancer risk in a Turkish population. Curr Med Res Opin 2011, 27:1295-1302

52. Yuan T, Wei J, Luo J, Liu M, Deng S, Chen P: Polymorphisms of base-excision repair genes hOGG1 326cys and XRCC1 $280 \mathrm{His}$ increase hepatocellular carcinoma risk. Dig Dis Sci 2012, 57:2451-2457.

53. Okasaka T, Matsuo K, Suzuki T, Ito H, Hosono S, Kawase T, Watanabe M, Yatabe Y, Hida T, Mitsudomi T, Tanaka H, Yokoi K, Tajima K: hOGG1 Ser326Cys polymorphism and risk of lung cancer by histological type. J Hum Genet 2009, 54:739-745.

doi:10.1186/1746-1596-9-108

Cite this article as: Peng et al:: Association between OGG1 Ser326Cys and APEX1 Asp148Glu polymorphisms and breast cancer risk: a meta-analysis. Diagnostic Pathology 2014 9:108. 